\title{
Ion Microscopy and Tomography
}

\author{
T. Butz, D. Lehmann, T. Reinert, D. Spemann and J. Vogt \\ Universität Leipzig, Fakultät für Physik und Geowissenschaften \\ Linnéstr. 5, 04103 Leipzig, Germany
}

\begin{abstract}
The use of light ions for microscopy and tomography is illustrated by a variety of recent applications in materials and life sciences at the Leipzig high-energy ion-nanoprobe LIPSION with a short comparison to other microscopic techniques. The versatility of ion techniques is exemplified by Rutherford backscattering spectrometry maps of thin films of solar cell materials, particle induced X-ray emission maps of manganese in ancient human bones, particle induced X-ray emission on single aerosol particles with $\mu \mathrm{m}$ size, and scanning transmission ion microscopy and tomography on pigs knee cartilage. Finally, the design of a single ion single living cell bombardment facility is sketched for studies of the cellular response and microdosimetry.
\end{abstract}

PACS numbers: 41.75.Ak, 72.20.My, 29.30.Kv

\section{Introduction}

The resolution of conventional optical microscopy is limited by the photon wavelength around $1 \mu \mathrm{m}$. Photons do not penetrate into the sample unless it happens to be transparent. The contrast is provided by the wavelength-dependence of the absorbance/reflectivity. There is "chemical blindness", i.e. the chemical constitution is not obtained by optical microscopy. A natural extension is the use of $\mathrm{X}$-rays, preferably obtained from synchrotron sources with a tunable wavelength. Such X-ray microscopes with resolutions in the order of $100 \mathrm{~nm}$ became feasible through the recent progress in X-ray optic devices. The penetration of X-rays is good, the generally poor contrast due to absorption can be outweighted by tremendous accuracy and, above all, by comparing images below and above absorption edges. In this way, the "chemical blindness" is overcome at the expense of sequential imaging at various wavelength. In principle, a full near edge and extended X-ray absorption fine structure (NEXAFS, EXAFS) spectrum could be recorded at each pixel thus allowing "chemical speciation". Drawbacks thus far are the large experimental effort and the lack of tunable X-ray lenses. 
Using massive charged particles like electrons overcomes several of these difficulties. The optical elements in electron microscopes are very advanced and rely on electromagnetic interactions of charged particles in vacuum. At sufficiently high energy the de Broglie wavelength is short enough to allow for atomic resolution. High energy electrons do penetrate into the sample, but their lateral straggling is severe and ultrathin samples are required for ultrahigh resolution. Thus, tomography is not really feasible with electrons. The excitation of characteristic X-rays provides chemical sensitivity - simultaneously for practically all elements! A disadvantage is the large Bremsstrahlungs background which impairs the detection limits. The use of even heavier particles like protons provides good penetration, small lateral straggling compared to electrons, and chemical sensitivity with very low detection limits due to the low Bremsstrahlungs background. These are the main reasons to develop ion microscopy and tomography despite the fact that the generation of $\mathrm{MeV}$ proton beams is slightly more expensive than the generation of $\mathrm{MeV}$ electron beams, true also for ion optical devices, and despite the fact that beam diameters below $1 \mu \mathrm{m}$ are difficult to achieve. The world record for the smallest $2 \mathrm{MeV}$ proton beam is at $40 \mathrm{~nm}$ diameter held by the Leipzig high-energy ion-nanoprobe LIPSION [1], still far above atomic resolution. However, it is the versatility of the ion beam techniques which renders ion microscopy and tomography an invaluable tool.

\section{Ion beam techniques, microscopy, and tomography}

Rather than to give an introduction to all possible ion beam techniques the reader is referred to the following books: Materials Analysis using a Nuclear Microprobe by Breese et al. [2], Applications of Nuclear Microprobes in the Life Sciences by Llabador and Moretto [3], and Particle Induced X-Ray Emission Spectroscopy (PIXE) by Johansson et al. [4]. For the present purpose it suffices to illustrate and discuss the most important techniques shown in Fig. 1 with particular emphasis on scanned ion beams in order to produce images as shown in Fig. 2.

$R B S$ : Rutherford backscattering spectrometry relies on the elastic scattering of light ions like protons or alpha particles from target atoms. The ions lose energy due to elastic collisions during their track into the sample and on the way out. The energy loss is measured and depends on the mass density of the target and on kinematics. Depth sensitivity is obtained via the stopping power of the target material. RBS maps are ideally suited for the investigation of the composition and homogeneity of the thickness of thin films.

$E R D A$ : elastic recoil detection analysis relies on the detection of matrix atoms recoiling after ion collisions rather than detecting backscattered projectiles like in RBS. Thus using $\mathrm{d} E / \mathrm{d} x$ and $E$-detectors a simultaneous multielement analysis is possible. The special case of protons as projectiles and protons (hydrogen) in the target as ejectiles offers the advantage of coincidence detection of both particles under 90 degrees, i.e. with very low background. 


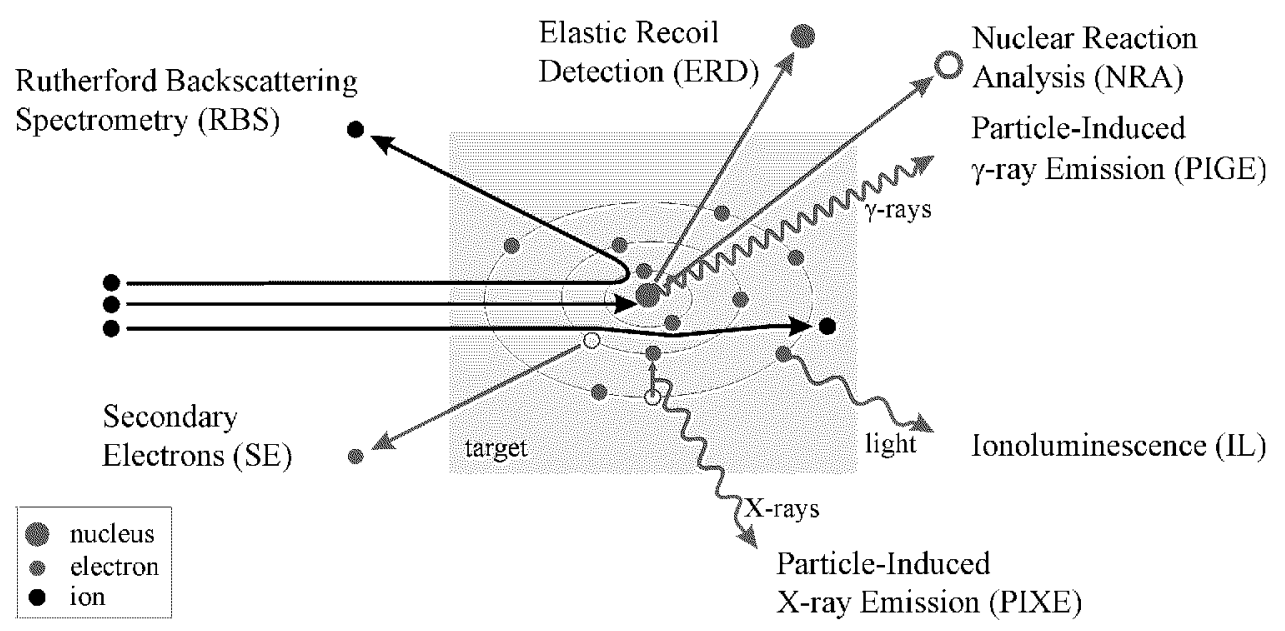

Fig. 1. Survey over common ion beam techniques showing projectile, target atom (nucleus and electrons), ejectiles, and radiation.

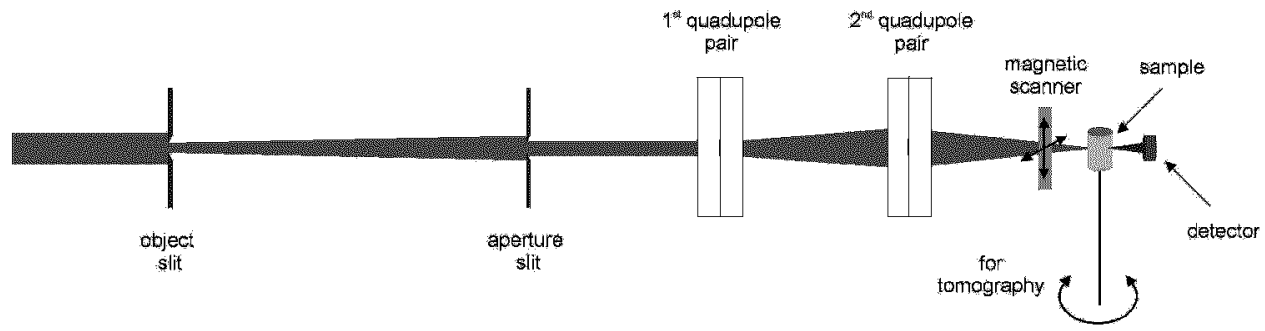

Fig. 2. Principle of ion microscopy and tomography.

PIXE: particle induced X-ray emission relies on the measurement of characteristic X-rays excited by particle bombardment of atoms. A simultaneous multielement analysis is possible which is essentially standard-free. Detection limits in the $0.5 \mu \mathrm{g} / \mathrm{g}$ range are possible. PIXE-maps are an indispensible tool for the investigation of lateral inhomogeneities of stoichiometries. Unfortunately, the depth resolution is poor and - even worse - the information depth is energy dependent due to the absorption of soft $\mathrm{X}$-rays in the target. Therefore, a combination of RBS, scanning transmission ion microscopy (STIM) and PIXE is often the strategy of choice.

PIGE and NRA: particle induced gamma emission and nuclear reaction analysis rely on the observation of characteristic $\gamma$-rays and particles, respectively, emitted from unstable nuclei or ejectiles produced in nuclear reactions. These techniques are particularly useful for threshold or narrow resonance reactions addressing a specific reaction at a time. Isotope specific images are obtained.

STIM: scanning transmission ion microscopy relies on the measurement of the energy of ions transmitted through the sample. Contrary to all other techniques 
discussed here there are no reaction cross-sections involved in this techniques and every single particle can be detected with nearly $100 \%$ efficiency. Thus $5-7$ particles per pixel are usually sufficient to obtain good quality images. Mass-density projections are generated in this way. If the sample is rotated in front of the scanned beam, a series of projections is obtained from which the 3-dimensional image can be reconstructed. A standard algorithm is the filtered Radon-backprojection.

$I L$ : ionoluminescence is related to the well-known cathodoluminescence. With modern ultrasensitive wavelength-sensitive detection devices light emitted under ion bombardment can be used to detect trace elements in specific chemical compounds. However, quantitative analysis is difficult because there are many - often unknown - mechanisms which activate or quench light emission and spectral bands are often wide and overlapping.

$S E I$ : secondary electron imaging relies on the detection of secondary electrons ejected under ion bombardment. The yield depends on the material and on the surface properties. Morphological images are obtained easily but are not always easy to interpret.

Summing up, there are two categories of ion beam techniques: "high-current" techniques like RBS, PIXE, PIGE, IL, SEI with typical currents in the range of 10-100 pA where radiation damage, particularly for biological samples, is an issue to be considered; "low-current" techniques like STIM or tomography with typical currents of the order of a few fA (or a countrate in the detector of several $\mathrm{kHz}$ for singly charged ions).

An important fact to mention is that the size of the beam spot in the limit of a small convergence angle is proportional to the square root of the spread in the beam energy and the chromatic aberration coefficient of the lens system and to the fourth root of the emittance [5]. Whereas the first two quantities can be optimized in the accelerator and lens designs, there is less freedom in optimizing the latter. The emittance is proportional to the beam current divided by the brightness. Since the reduced brightness, i.e. the brightness divided by the ion energy, is limited by the ion source, the only way to achieve ultra-high resolution is by reducing the beam current drastically.

The Leipzig high-energy ion-nanoprobe LIPSION ("lipsia" is the latin and italian - word for linden tree where the name Leipzig came from, and "ion" is self-explanatory) was designed to achieve ultra-high resolution far below that of conventional optical microscopes, an indispensible requisite for keeping pace with the rapidly developing X-ray microscopes. A 3.5 MV ultra-stable SINGLETRON by high voltage electrical engineering (HVEE) with an RF ion source for protons and alphas is coupled to a Melbourne nuclear microprobe with a split Russian quadruplet with a demagnification in $x$ and $y$ of 130 . The ripple is below $30 \mathrm{~V}_{\mathrm{pp}}$ at $2.25 \mathrm{MV}$, the voltage and analysing magnet stability are better than $10^{-5}$ and great care was taken to reduce mechanical vibrations as much as possible. Up to now we have achieved about $300 \mathrm{~nm}$ lateral resolution for PIXE and about $80 \mathrm{~nm}$ 


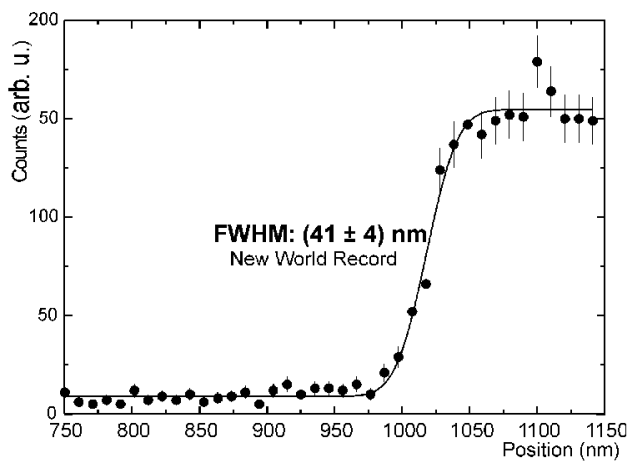

Fig. 3. Scan of ion beam over atomically sharp InGaP/GaAs semiconductor edge in STIM-mode showing beam diameter of $40 \mathrm{~nm}$.

for STIM. In fact, the beam diameter is $40 \mathrm{~nm}$ only, as measured by a line scan over an atomically sharp semiconductor edge (see Fig. 3). At present, quasi-stationary magnetic stray fields deteriorate the resolution. We hope to achieve STIM images with a resolution better than $40 \mathrm{~nm}$ using an active compensation of the stray fields in the near future.

\section{Examples}

In the following section a few typical examples of recent activities at LIPSION are given to illustrate the power of ion microscopy and tomography. This is a rather narrow view and the reader is referred to the review books [2-4] for a broad overview.

\subsection{Thin films of $Z n_{2-2 x} C u_{x} I n_{x} S_{2}$}

Thin films of $Z_{2} n_{2-2 x} \mathrm{Cu}_{x} \mathbf{I n}_{x} \mathrm{~S}_{2}$, a promising solar cell material, were produced on various substrates by pulsed laser deposition (PLD). The following fundamental questions arise: (i) does the target stoichiometry translate to the thin film and, if so, how is the homogeneity? (ii) is the film thickness homogeneous? (iii) what is the morphology (grain size, texture etc.)? (iv) what are the laterally resolved electrical properties? The last question can be answered efficiently by ion beam induced charge collection (IBIC) on contacted films, preferably also in a time-resolved mode [6]. An IBIC-facility is presently under construction at LIPSION. The morphology is usually studied by X-ray diffraction and high resolution electron microscopy (HREM). To answer the first two questions RBS and PIXE are the methods of choice. In Fig. 4 (top left) a $41 \mu \mathrm{m} \times 41 \mu \mathrm{m} 2 \mathrm{MeV}$ alpha-RBS-map is shown for a thin film with a nominal thickness of about $200 \mathrm{~nm}$. Obviously, the map looks rather inhomogeneous. This could be either due to inhomogeneities in the stoichiometry or due to a variable film thickness. On the right, the "broad 


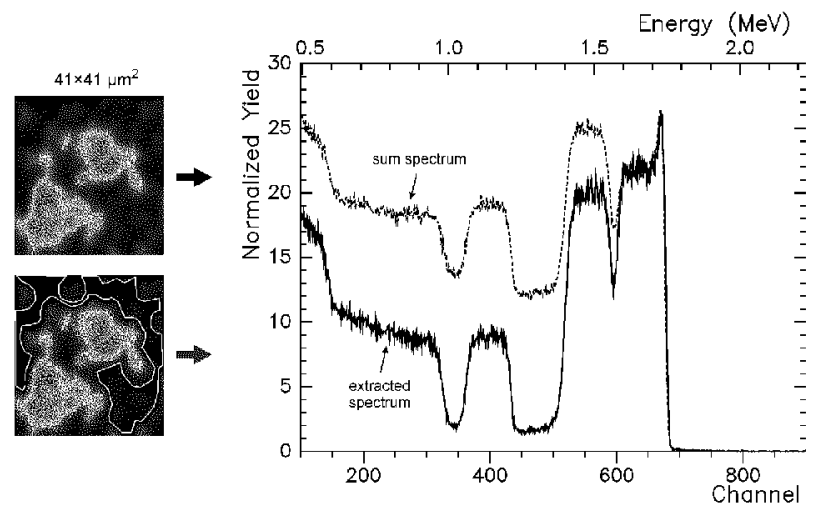

Fig. 4. Alpha-RBS-map (left top) of a thin film of $\mathrm{Zn}_{2-2 x} \mathrm{Cu}_{x} \mathrm{In}_{x} \mathrm{~S}_{2}$ showing a rather inhomogeneous pattern; right: RBS spectrum of this area, called "sum spectrum" (dashed) and the area selected as shown in the left bottom map (solid).

field" RBS-spectrum is shown which is calculated from the map as the "sum spectrum". This alone would be rather difficult to interpret: the individual layers of In and $\mathrm{Cu} / \mathrm{Zn}$ (which cannot be separated) are clearly visible (according to $Z$ from right to left) but there is substantial intensity in between the In-region and the $\mathrm{Cu} / \mathrm{Zn}$-region where there should be none. The same holds true for the gap between the $\mathrm{Cu} / \mathrm{Zn}$-region and the S-onset. It is now possible - like in a jigsaw puzzle - to cut out regions which look relatively homogeneous (see Fig. 4 left bottom) and display the RBS-spectrum of this area alone. This results in a much improved spectrum from which the thickness of this area can be derived accurately to be $230 \mathrm{~nm}$. From PIXE-maps, not shown here, we concluded that the stoichiometry of this area and that of the left-over "islands" is about the same. Thus the "islands" are indeed "rocks" of $\mu \mathrm{m}$ dimensions which very likely were sputtered as a whole by the pulsed laser deposition (PLD) process. This clearly points to an inadequate target preparation process. Needless to say that this example does not represent the present state of the art of producing PLD-films but was chosen for illustrative purposes only.

\subsection{Trace elements in ancient human bone}

The determination of main and trace elements in ancient human bone serves as a sort of diary for anthropologists from which information on eating habits and diseases can be derived. However, postmortem diagenetic alterations may corrupt this "diary" and it is of utmost importance to learn to what extent ions have been leached out of the bone or incorporated into the bone by mineral exchange processes or microbial influences in the burial ground. Spatially resolved IL-data on samples from a Merowingian population near Frankfurt/Main, Germany, showed that there is an intense orange luminescence in the periostal regions of the ancient bone which fades out after a few $\mathrm{mm}$ and turns over into a blue 
luminescence, like that observed for recent bones throughout the sample [7]. The suspicion was that Mn-ions are incorporated into hydroxylapatite and we therefore took $84 \mu \mathrm{m} \times 84 \mu \mathrm{m}$ maps of a region close to a pore in the bone sample. Figure 5

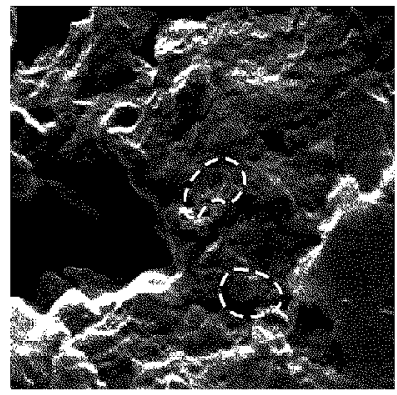

SE

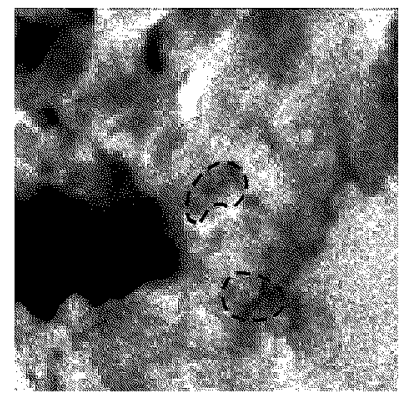

$\mathrm{Ca}$
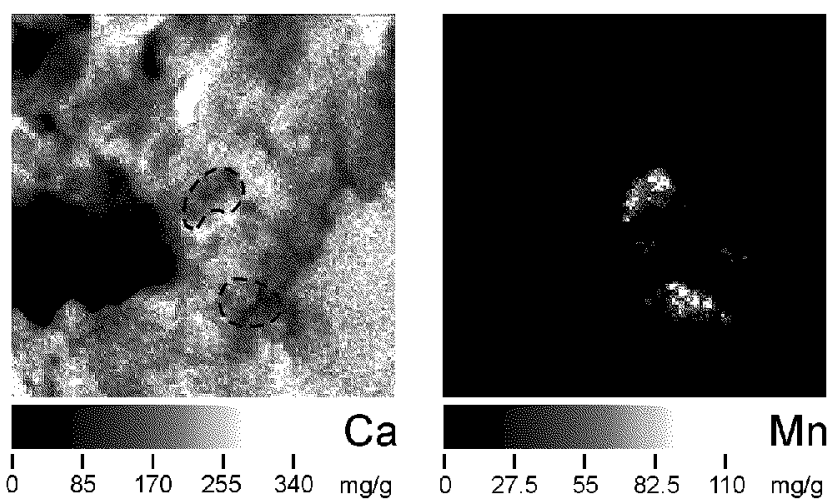

Fig. 5. From left to right: SE-map, Ca-map, Mn-map of a sample of ancient human bone.

(left) shows an SE-map which closely resembles the Ca-map (middle) and gives an idea of the morphology. The Mn-map (right) shows two pronounced spots surrounded by clouds with unusually high Mn-concentration. However, if integrated over large areas the slight increase in the average Mn-concentration might have been overlooked with less sensitive methods than IL. At present we cannot decide whether Mn was incorporated via mineral exchange through pores or whether it is a result of the remnants of microbe colonies. In any case, the Mn-rich zone should not be considered a "virgin diary". Correlations between several trace elements indicate that in fact this outermost zone is certainly contaminated by various elements and should not be considered useful for further interpretations.

\subsection{Single aerosol particles}

The analysis of the composition of aerosol particles is usually carried out collecting a large amount of particles in a compactor and determining the average composition. This is rather unsatisfactory if the sources of pollution are rather diverse. In order to trace back the source of pollution the identification of individual aerosol particles — often as small as $1 \mu \mathrm{m}$ and below — is necessary. This can be achieved by $\mu$-PIXE. In Fig. 6 a PIXE-map $(8 \mu \mathrm{m} \times 8 \mu \mathrm{m})$ of two aerosol particles from Shanghai is shown together with their spectra from which - using a fingerprint "library" established by a neural network - the type of source can be identified unambiguously: the left particle comes from a blast furnace, the right one from a cement factory [8]. 

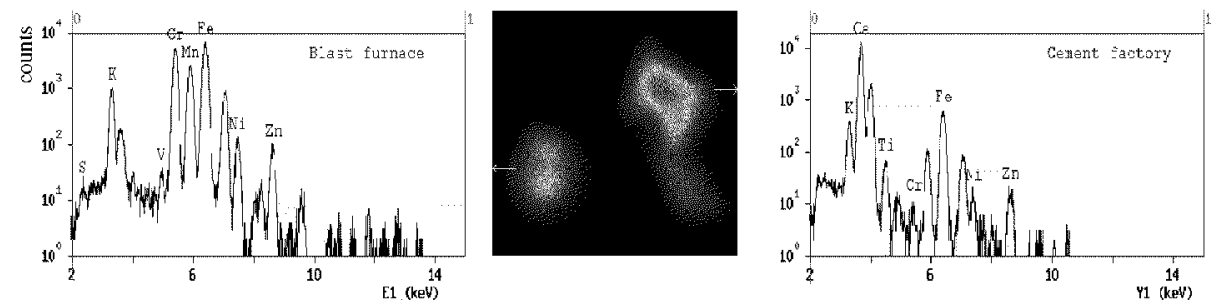

Fig. 6. PIXE-map of two aerosol particles of different provenance in the Shanghai area. The insets show characteristic PIXE-spectra used as fingerprints for identification.

\subsection{Cartilage}

The degradation of cartilage in joints lead to a very common and painful disease: arthrosis. At present we have no good means for an early diagnosis nor is there a good therapy. The metabolism in cartilage proceeds via kneading (like a sponge) since there are no vessels. Hence, the knowledge of the internal architecture of cartilage is of crucial importance to understand its functioning and malfunctioning. Cartilage is by no means a homogeneous substance. Under an optical microscope cartilage cells, called chondrocytes, with typical dimensions of $10 \mu \mathrm{m}$ are easily visible. In some areas they appear as isogonic pairs with a characteristic orientation. This immediately calls for the hypothesis that the collagen fibres are aligned in the same way. This is in fact borne out by electron microscopy

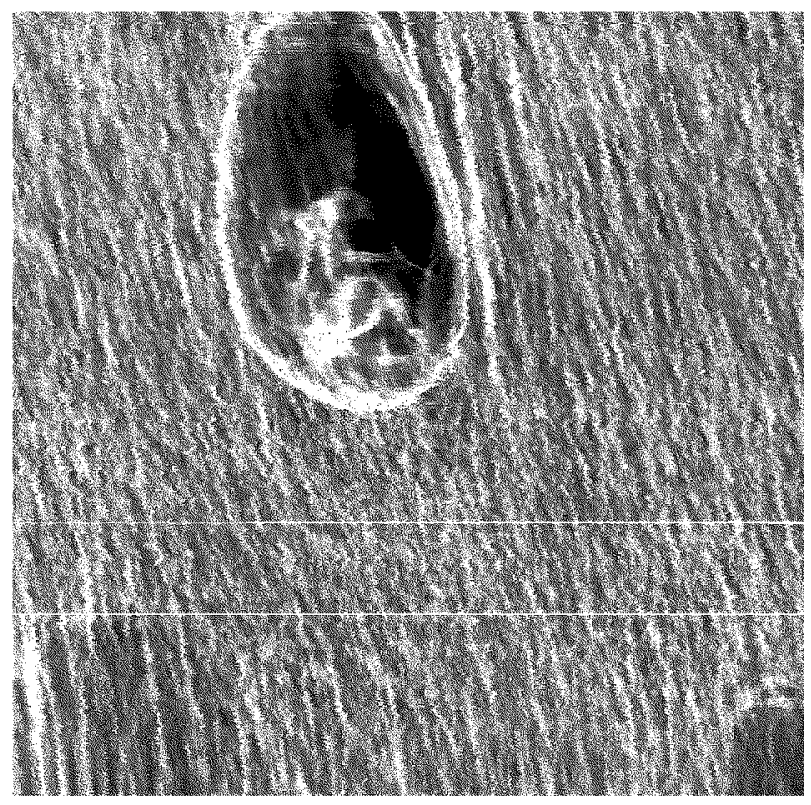

Fig. 7. STIM-image $(40 \mu \mathrm{m} \times 40 \mu \mathrm{m})$ of a thin slice of cartilage from a pigs knee showing a chondrocyte and the well aligned collagen fibres (white). 
on freeze fractured samples and was deduced as well from angle resolved nuclear magnetic resonance microscopy [9]. These collagen fibres are too small to be observable by optical microscopy, but are easily seen in ion-microscopy. Figure 7 shows a $40 \mu \mathrm{m} \times 40 \mu \mathrm{m}$ STIM image of a sample from a pigs knee cartilage with a lateral resolution of $80 \mathrm{~nm}$. The sample was freeze dried. The chondrocyte and intracellular structures - difficult to interpret due to the freeze drying - are clearly visible, as are the highly oriented collagen fibres [10]. It turns out that the type and degree of orientation as well as the micro-architecture of the collagen fibres varies drastically over various regions of the tibial and femoral cartilage. PIXE-maps, not shown here, exhibit $\mu \mathrm{m}$-spots of high Ca-concentration which are not paralleled by $\mathrm{P}$-spots. Their origin is not yet clear but these might be nuclei for solid particle formation which mechanically deteriorate the cartilage upon kneading. Further studies on cartilage with and without mechanical load will elucidate the transport of nutrients and metabolic products.

Finally, first ion tomographic images were obtained on a cartilage sample of dimensions $10 \mu \mathrm{m} \times 28 \mu \mathrm{m} \times 30 \mu \mathrm{m}$. Projections were collected every 0.5 degree for 180 degrees. Since the sample had no resemblance to a cylinder (like that shown in Fig. 2) the sinograms had to be corrected substantially using the relatively sharp contours of the chondrocyte as a sort of "fiducial marker". Figure 8 shows the reconstructed image which - unfortunately - cannot rotate on a printed paper. The big advantage of ion tomography compared to electron microscopy is that images of "thick" samples (say $30 \mu \mathrm{m}$ tissue) can be obtained in a non-destructive manner. We hope to improve the resolution in our tomography such that individual

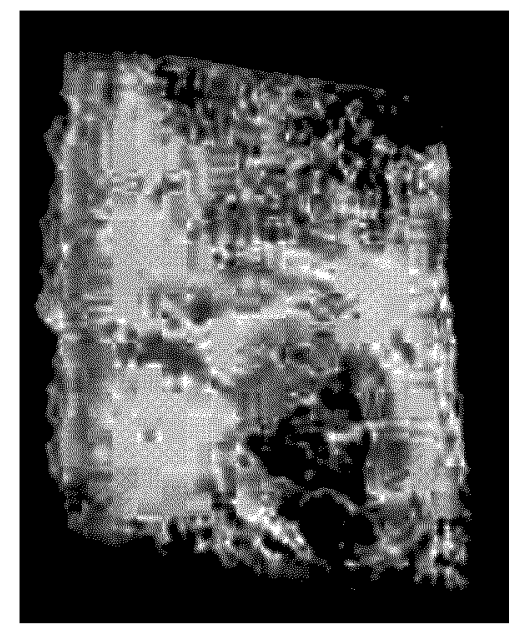

Fig. 8. Perspective view of a cartilage slice $(28 \mu \mathrm{m} \times 10 \mu \mathrm{m} \times 30 \mu \mathrm{m})$ obtained by ion tomography. Grey represents high density material (large energy loss), light grey represents medium density material. The low density material was omitted for the sake of clarity. 
collagen fibres or eventually collagen tubes are directly visible. Tomography with an external beam would be highly desirable because the freeze drying procedure could be avoided and experiments in which the pressure on cartilage samples can be cycled would become feasible.

\section{Outlook: single ion bombardment of single living cells}

When counting individual particles like in STIM, when talking about external beams, and when dealing with biological material the question of targeted bombardment of a single living cell by an exactly counted number of particles comes up naturally. A single alpha particle with a few $\mathrm{MeV}$ traversing a cell nucleus can produce a measurable damage. A hot topic is the so-called bystander effect: a cell is hit and some of the bystanding cells respond as if they were hit, too. Questions of microdosimetry can be addressed with completely new dimensions. Consequences for radiotherapy are foreseeable. Up to now, collimated external ion beams are in use with roughly $1 \mu \mathrm{m}$ lateral accuracy. Focused external ion beams with much better resolution and single living cell bombardment facilities are presently under construction at various places in the world [11]. A sketch of the facility at LIPSION is shown in Fig. 9. Cells are cultivated on a mini-Petri dish and the coordinates of the nuclei are recorded in a cell-observer. These dishes are then brought to the irradiation platform and mounted upright. Cells which adhere sufficiently to the mylar bottom of the dish retain their positions for a couple of minutes, as has been tested earlier. A fiducial marker on the mini-Petri dish - not

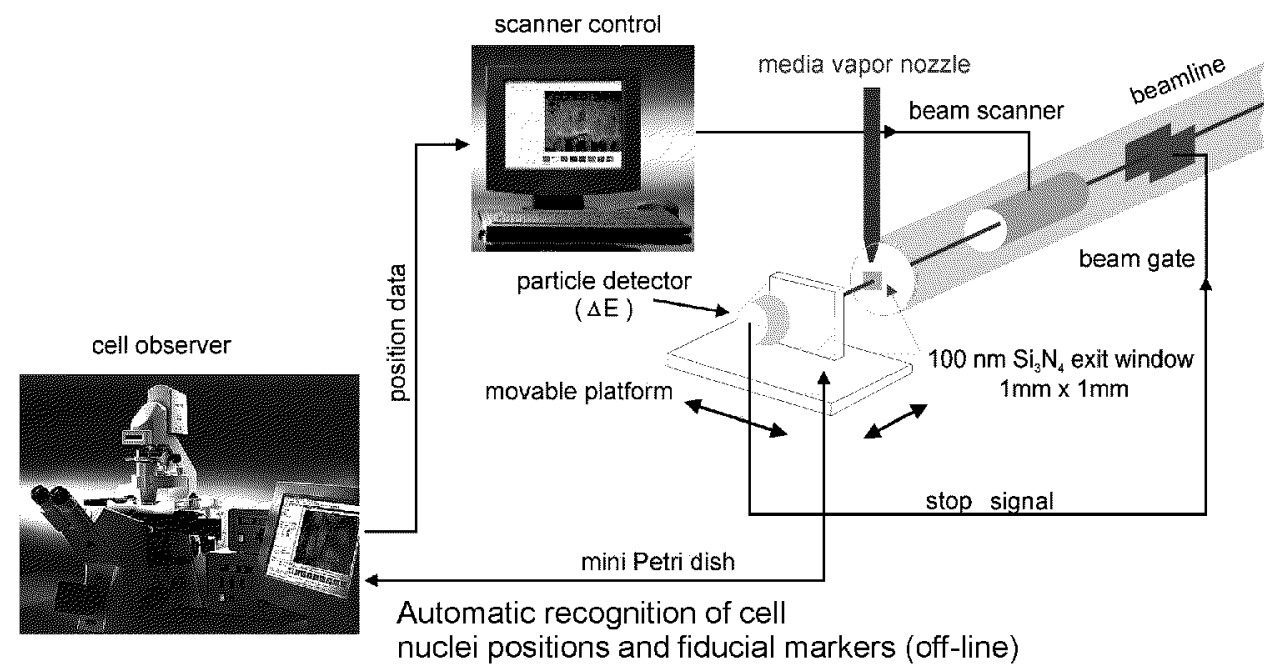

Fig. 9. Sketch of the single ion single living particle bombardment facility at LIPSION (under construction). 
in contact with the media - helps to precisely position the dish via STIM. The coordinates of the cell nuclei are transferred to the scanner and a single (or more) selected cell nucleus (or cytoplasm) is bombarded with a preselected number of particles. A particle detector behind the cells triggers the beamgate upstream and registers the energy lost in the exit window, the mylar bottom of the mini-Petri dish, the cell, and the media. After bombardment we analyze the cell culture for micronucleus formation and apoptosis off-line.

\section{Acknowledgment}

It is a pleasure to thank our various collaborators: M. Gründer, U. Reibetanz (Institute of Medical Physics and Biophysics, University of Leipzig), J. Zhu (Shanghai Institute of Nuclear Research), St. Jankuhn (Institute for Surface Modification, Leipzig), J. Hammerl (Institute of Anthropology, University of Frankfurt/M.), A. Sakellariou (University of Melbourne), J. Tanner (Clinic of Radiation Oncology, Martin-Luther University Halle-Wittenberg). This work was supported by the Deutsche Forschungsgemeinschaft (Center of Excellence Program "Phänomene an den Miniaturisierungsgrenzen"; individual grant "Ionenmikroskopie").

\section{References}

[1] D. Spemann, T. Reinert, J. Vogt, D. Dobrev, T. Butz, to be presented at the 15 th Int. Conf. on Ion Beam Analysis, Cairns 2001, Australia.

[2] M.B.H. Breese, D.N. Jamieson, Ph.J.C. King, Materials Analysis Using a Nuclear Microprobe, Wiley, New York 1996.

[3] Y. Llabador, Ph. Moretto, Applications of Nuclear Microprobes in the Life Sciences, World Sci., Singapore 1998.

[4] Particle-Induced X-Ray Emission Spectroscopy (PIXE), Eds. S.A.E. Johansson, J.L. Campbell, K.G. Malmqvist, Wiley, New York 1995.

[5] G.J.F. Legge, J.S. Laird, L.M. Mason, A. Saint, M. Cholewa, D.N. Jamieson, Nucl. Instrum. Methods Phys. Res. B 77, 153 (1993).

[6] B.L. Doyle, G. Vizkelethy, K.M. Horn, D.S. Walsh, and P.E. Dodd, in: Proc. 16th Int. Conf. on the Application of Accelerators in Research and Industry, Denton (USA) 2000, CP567 AIP Press, 2001.

[7] D. Spemann, St. Jankuhn, J. Vogt, T. Butz, Nucl. Instrum. Methods Phys. Res. $B$ 161-163, 867 (2000).

[8] P. Guo, J. Wang, X. Li, J. Zhu, T. Reinert, J. Heitmann, D. Spemann, J. Vogt, R.-H. Flagmeyer, T. Butz, Appl. Spectrosc. 54, 807 (2000).

[9] W. Gründer, M. Kanowski, M. Wagner, A. Werner, Magn. Reson. Med. 43, 884 (2000).

[10] T. Reinert, U. Reibetanz, J. Vogt, T. Butz, A. Werner, W. Gründer, Nucl. Instrum. Methods Phys. Res. B, in press.

[11] $5^{\text {th }}$ International Workshop on Microbeam Probes of Cellular Radiation Response, Stresa (Italy) 2001, Radiation Protection and Dosimetry, in press. 